\title{
Analysis of parallel computing technology in cloud computing
}

\author{
LI Jun ${ }^{1, a}$ \\ ${ }^{1}$ School of Information Science and Technology, Chengdu University of Technology, Chengdu, \\ 610059, China \\ aemail: lijuncd@126.com
}

Keywords: Cloud computing; key technology; parallel computing

\begin{abstract}
Cloud computing is a new leading information technology, combined with the IT technology and the Internet, super computing and storage capacity, and its goal is to as water supply, power supply, the financial system, "calculation" as a kind of public infrastructure and organization of large-scale information and computational resources, provide a convenient, comprehensive public service for the user, to meet the needs of the personal and social information service.
\end{abstract}

\section{Introduction}

Cloud computing's original idea can be traced back to the 1960s, Turing Award winner John McCarthy mentioned: "in the near future, the calculation may become a kind of public infrastructure". By the end of 2007, IBM was the first to propose their cloud computing program, and cloud computing platform described as an on-demand dynamic deployment and configuration of scalable platform[1].

There is still no universally agreed definition of cloud computing. Definition of the National Institute of standards and technology of cloud computing, cloud computing is a model, the model is easily on-demand access to a common set of allocation of computing resources (for example, networks, servers, storage devices, applications and service). These resources can be quickly provided and published, while minimizing the cost of management or the interference of service providers. China Cloud Computing definition given by the expert Liu are as follows: cloud computing tasks are distributed to a large number of computers in the pool of resources, so that a variety of applications can access according to the need of computing power, storage space and a variety of software services.

Popular understanding, cloud computing cloud is from the cluster of servers on the Internet resources, including hardware and software resources, the local computer only need through the Internet to send a demand for information, it is possible to get the required computational resources.

Cloud computing development is inseparable from the virtualization, parallel computing and other core technology development mature is the computer technology and network technology development is a combination of cloud computing, and with the help of SaaS/PaaS/IaaS advanced business model to the powerful computing capability of distributed to the hands of the user terminal.

1) virtualization technology

Virtualization is the core feature of cloud computing, cloud computing is the basis of relying on. Virtualization technology to achieve the logical abstraction of physical resources and unified, it refers to the calculation of components on the basis of the virtual and not on the basis of real hardware. Through virtualization technology can achieve optimal resource utilization, and can according to the change of user business needs, on-demand resource allocation to achieve dynamic load balancing; also has nothing to do with hardware characteristics of the self-healing system function, system to enhance the reliability.

The object of virtualization technology can be covered from the server, storage, network to the platform, application and other aspects.

2) distributed technology

Distributed technology is the first to provide search services to the global user by the scale of 
Google, its distributed architecture, you can make up to one million units of low-cost computers to work together. Distributed file system to complete the data distributed storage, distributed computing programming model complete large task decomposition and parallel computing based on multiple computers, distributed database to complete massive structured data storage.

Distributed file system as a distributed technology is the most important part of the implementation of, whether it is Google GFS or Hadoop HDFS, are designed for a specific large file storage applications, can achieve high reliability of data storage and access, access to the high performance, the migration and automatic load balancing.

3) parallel computing

Parallel computing is one of the effective ways to solve the problem of computing resources and to improve the computing speed and processing power of computer systems. Its basic idea is to use multiple processors to solve the same problem, the problem is decomposed into several parts, and each part is calculated by an independent processor.

Parallel computing is relative to the serial calculation (usually for a single CPU or a single computer), it will process relatively independent distribution on different nodes, by the independent operating system scheduling, enjoy independent of CPU and memory resources (memory that can be shared); process between mutual information exchange via message passing.

$\mathrm{P}$ aaS/ I aaS 4.SaaS/ service model

Innovation of service mode is an important property of cloud computing, cloud computing the full realization of the computer software and hardware, is service changes, the user in the future need to things only one kind of service, including computing services, network services, software services, service platform, storage services. Cloud computing services are mainly: SaaS (software as a service), PaaS (platform that services) and IaaS (infrastructure, namely services).

SaaS is the user access to a new form of software services, users do not need to install software on their computer or server, and directly through the network to specialized suppliers to get the needed software services.

PaaS refers to the development environment (including application design, application development, application testing and application hosting) as a service provider to the customer. Customers do not need to buy hardware and software, only need to use the PaaS platform, you can create, test and deploy applications and services.

IaaS is a business or individual can use cloud computing technology to remote access to computing resources, including computing, storage and application virtualization technology provided by the relevant features. Both the end user and provider of SAAS and PAAS provider can from the infrastructure services to obtain the required computing power, but there is no need to pay the corresponding original investment cost of the computing capability of the IT infrastructure software and hardware support.

\section{Development status of cloud computing}

Cloud computing is a hotspot, in recent years all over the world about its research and application is in the ascendant, many government departments and the famous company in the research, development and application of cloud computing in the process made a lot of work and effort[2].

1) development of cloud computing in foreign countries

Cloud computing is inseparable from the network. The original meaning of cloud computing is to provide computing power through the Internet. The origin of cloud computing and the Amazon and Google two companies have very close relationship, they are the first to use the "Computing Cloud" means of expression. Currently the United States announced the development of the industry giants to enter or support cloud computing technology, including Microsoft, Google, IBM, Amazon, Netsuite, NetApp, Adobe, etc..

Le Goog is the proposed cloud computing. In 2006, Le Goog launched the "Google101" program to guide college students to carry out the "cloud" system programming. The accumulation of years of search engine technology achievements to Google in the cloud computing technology is in the 
leading position, not only to provide online application, also hope to play in its advantages of database system, a unified platform for online applications. Google to publish academic papers in the form of a public cloud computing three magic weapons: GFS, Map/Reduce and BigTable, and in the United States, China and other colleges and universities to open cloud computing programming.

Microsoft in October 2008 launched the Azure Windows operating system, the system as a server side cloud computing program operating system (OS Cloud) for the majority of developers to provide services. Microsoft has hundreds of millions of the world's Windows user desktop and browser, azure (blue) tried to through the architecture of the Internet to create a new cloud computing platform, let the wind ows by PC extends to the "blue sky"[3].

IBM from the gradual rise of internal demand, in November 2007 proposed the blue cloud program, launched a total of cloud and private cloud concept. IBM proposed private cloud solution is to reduce the existing problems such as data, information security, and so on, to seize the enterprise cloud computing market. Relying on the traditional advantages of IBM in the field of the server, IBM become the only one available from hardware, software to all the independent production of the manufacturer.

In July 2008, Yahoo!, Hewlett Packard and Intel jointly announced will establish a global open source cloud computing research test bed, called open cirrus, encourage research in various aspects of the development of cloud computing, service and data center management field.

Apple is an active participant in the field of cloud computing. From the recent launch of the iTunes service to mob ile me service, to the acquisition of online music service providers Lala, to recently in the United States, North Carolina investment 10 billion plan to establish the new data center, all without exception shows its foray into cloud computing in the field of great determination.

These well-known international companies in the world to build a huge cloud computing center. For example: Le Goog search engine distributed in more than 200 sites, more than 1 million server support, and the number of facilities is growing rapidly.

2) the development of cloud computing in China

At present, most of China's cloud computing focuses on the concept, technology and model of early cloud computing. Early cloud computing is a dynamic, easy to expand, through the Internet to provide a virtual IT resources and application of a computing model. Users do not need to understand the details of the cloud technology, do not have the professional knowledge of the cloud, but not directly involved in investment, construction, maintenance and control will be able to directly use and according to the amount paid.

2008, IBM established China's first cloud computing center in Beijing, IBM China Innovation Center in Wuxi to establish a second Cloud Computing Center - IBM Greater China Cloud Computing center. In early 2009, the establishment of the first e-commerce cloud computing center in Nanjing". Century Internet launched the "CloudEx" product line, including the complete Internet hosting service "Comp uting Service CloudEx", based on online storage virtualization "Storage Service CloudEx" and other cloud computing services.

With rising of cloud computing, the domestic telecom operators are also actively into the cloud computing research, in order to through the cloud computing technology to promote the optimization and integration of the network structure, to find new profit opportunities and profit growth point, in order to achieve the transformation of the information service enterprises. China Mobile launched the "cloud" (big cloud cloud based service platform, China Telecom launched the "e" cloud in cloud computing platform, China Unicom is to launch the Internet cloud platform.

Our country enterprise created "cloud security" concept, through a large network of clients on the network behavior anomaly detection, access to the Internet Trojans, and malicious program of the latest information, automatic analysis and processing on the server, then the solutions to distribute to the client. The rising trend, and other companies have launched cloud security solutions.

With the development of cloud computing, the Internet has become more and more powerful, 
and users can process large data and obtain the required information on the Internet. From the development situation of cloud computing, the development of cloud computing in the future will be able to build a large scale and the application of the underlying infrastructure in the direction of development. Constantly create new cloud computing applications, to provide users with more and more sophisticated Internet services can also be used as a development direction of cloud computing.

\section{Cloud computing and distributed computing}

Cloud computing is virtual, infrastructure as a service, platform as a service, software as services, such as the concept of hybrid evolution and jump results, is also a distributed computing, grid computing and parallel computing, the latest development, or commercial implementation of these computer science concepts. Distinguish between the different forms of the difference, will help us to understand and grasp the essence of the cloud computing.

1) cloud computing and distributed computing

Distributed computing is a term in a loosely or tightly controlled conditions using a hardware and software system for processing tasks, the system contains multi processor unit or a storage unit, a plurality of concurrent process and procedures. A program is divided into several parts, and runs on a computer connected to the network. Distributed computing is similar to parallel computing, but parallel computing is often used to refer to a number of parts of a program running on a number of processors on a single computer. So, distributed computing usually must deal with heterogeneous environment, a variety of network connection, unpredictable network or computer error. It is clear that the cloud computing belongs to the category of distributed computing, and it is a distributed computing mode which is oriented to the foreign service. Cloud computing applications and systems based on the cheap large-scale server clusters, by infrastructure and upper layer application can cooperate to build in order to achieve maximum efficiency utilization of hardware resources and through the method of software tolerance error of a plurality of nodes to distributed computing system scalability and reliability goals.

2) cloud computing and grid computing

If you simply according to the definition of grid grid will be the high-speed Internet, high-performance computer, large databases, sensors, remote equipment integration, for users to provide more resources, function and service, between cloud computing and grid computing, it is difficult to distinguish. But at present some mature cloud computing examples, cloud computing and grid computing have some differences. Grid computing emphasizes is a composed of multi institutional virtual organizations, and multiple agencies of different servers constitute a virtual organization to provide users with a powerful computing resources; Cloud Computing mainly uses virtual machines (virtual server) polymerization and the formation of homogenous service, more emphasis on the sharing of distributed computational resources within an organization. In the grid environment can not be huge computing program split into numerous smaller subroutines in multiple agencies provide resources between the treatment, and in the cloud calculation environment because of the resources to ensure that the user running environment, the user submits a handler solution into smaller subroutines in the different resources for processing becomes possible research and development. In the business model, job scheduling, resource allocation, whether to provide services and their forms, the difference is obvious[4].

3) cloud computing and parallel computing

In simple terms, parallel calculation is in parallel computer calculation, it and often said that the high performance computing, super computing is synonymous with, because any high performance computing and supercomputing is inseparable from the overall parallel technology. Parallel computing is evolved on the basis of serial computing, it is hard to simulate the natural world, a sequence containing a large number of simultaneous, complex and related events in the state of affairs. In recent years, with the continuous development of hardware technology and new applications, parallel computing also has a number of new developments, such as multi-core architecture, cloud computing, personal computer, etc.. So, cloud computing is a form of parallel 
computing, and it also belongs to one of the forms of high performance computing and super computing. As the parallel computing of the latest development of computing model, cloud computing means for server-side parallel computing requirements is enhanced, because the application of tens of thousands of users is achieved through the Internet in the cloud, it brings users and business model fundamentally changing, on massively parallel computing technology proposed new requirements[5].

\section{Conclusion}

Cloud computing is a new computing model based on a variety of technologies, the globalization, information and automation of modern software applications and business processes, and will provide a wide range of market and application background for the research and development of cloud computing. Cloud computing has the characteristics of super large scale, virtualization, high availability, high reliability, on-demand service and its cheap, after many successful cases and experiences in recent years, it is widely used. Cloud computing brings new IT resources sharing and application mode is another change after the Internet, will give the IT industry, especially the software service industry has far-reaching impact.

\section{References}

[1] Satoshi Kagami,Tomonobu Kitagawa,Koichi Nishiwaki,Tomomichi Sugihara,Masayuki Inaba, Hirochika Inoue. A Fast Dynamically Equilibrated Walking Trajectory Generation Method of Humanoid Robot [J], 2002.

[2] HIROKIK, MINORUA, YASUOK, ET al.RoboCup: a challenge problem for AI and robotics. HirokiK. RoboCup-97: Robot Soccer World Cup [C]. Berlin: Springer, 1998.38-43.

[3] Zhizhong Yin. Application of FPGA control DC motor servo system [J]. Inner Mongolia Science and Technology and Economy, 2008177 (23) 101-103.

[4] Joerg Christian Wolf, Phil Hall, Paul Robinson, Phil Culverhouse. Bioloid based Humanoid Soccer Robot Design, 2007.

[5]Wu Chuan-yu, He Lei-ying, Design and Realization of Instructional RPPR-Robot, Research and Exploration in Laboratory.2007, 26(10). 